Article

\title{
Fine Physical Bin Mapping of the Powdery Mildew Resistance Gene Pm21 Based on Chromosomal Structural Variations in Wheat
}

\author{
Shanying Zhu ${ }^{1,2}$, Yaoyong $\mathrm{Ji}^{1}$, Jian $\mathrm{Ji}^{1}{ }^{1}$, Tongde Bie ${ }^{3}$, Anli Gao ${ }^{4, *}$ and Huagang $\mathrm{He}^{1, *}$ \\ 1 School of Food and Biological Engineering, Jiangsu University, Zhenjiang 212013, China; \\ zhushanying@mail.ujs.edu.cn (S.Z.); 2211618014@stmail.ujs.edu.cn (Y.J.); jijian221118005@163.com (J.J.) \\ 2 School of Environment, Jiangsu University, Zhenjiang 212013, China \\ 3 Yangzhou Academy of Agricultural Sciences, Yangzhou 225007, China; btd@wheat.org.cn \\ 4 School of life Sciences, Henan University, Kaifeng 475004, China \\ * Correspondence: algao@henu.edu.cn (A.G.); hghe@mail.ujs.edu.cn (H.H.); \\ Tel.: +86-371-23887799 (A.G.); +86-511-88780201 (H.H.)
}

Received: 31 January 2018; Accepted: 22 February 2018; Published: 24 February 2018

\begin{abstract}
Pm21, derived from wheat wild relative Dasypyrum villosum, is one of the most effective powdery mildew resistance genes and has been widely applied in wheat breeding in China. Mapping and cloning Pm21 are of importance for understanding its resistance mechanism. In the present study, physical mapping was performed using different genetic stocks involving in structural variations of chromosome 6VS carrying Pm21. The data showed that 6VS could be divided into eight distinguishable chromosomal bins, and Pm21 was mapped to the bin FLb4-b5/b6 closely flanked by the markers 6VS-08.6 and 6VS-10.2. Comparative genomic mapping indicated that the orthologous regions of FLb4-b5/b6 carrying Pm21 were narrowed to a $117.7 \mathrm{~kb}$ genomic region harboring 19 genes in Brachypodium and a $37.7 \mathrm{~kb}$ region harboring 5 genes in rice, respectively. The result was consistent with that given by recent genetic mapping in diploid $D$. villosum. In conclusion, this study demonstrated that physical mapping based on chromosomal structural variations is an efficient method for locating alien genes in wheat background.
\end{abstract}

Keywords: Pm21; powdery mildew resistance; physical mapping; comparative mapping

\section{Introduction}

Common wheat (Triticum aestivum L.) is one of the most important cereal crops. Wheat production is seriously threatened by powdery mildew caused by Blumeria graminis f. sp. tritici (Bgt). The powdery mildew resistance gene Pm21, originating from Dasypyrum villosum Candargy ( $2 n=14, \mathrm{VV})$, confers highly effective resistance to all known isolates of $B g t[1,2]$. As the donor of Pm21, wheat- $D$. villosum translocation line T6AL.6VS has been widely applied in wheat breeding. More than 20 varieties with this translocated chromosome have been planted on an accumulated area of more than four million hectares in China [3]. However, up to now, little is known about the nature of Pm21 and its resistance mechanism. Therefore, it is essential to map and clone Pm21.

Previously, Pm 21 had been mapped to the chromosomal bin FL0.45-0.58 of 6 VS using two deletion lines del.6VS-1 (FL0.58) and del.6VS-2 (FL0.45) [2,4]. In the recent study, 6VS FL0.45-0.58 was verified to be closely flanked by the markers 6VS-03 and 6VS-23 via physical mapping and comparative mapping, which narrowed the orthologous regions of FL0.45-0.58 to $1.06 \mathrm{Mb}$ in Brachypodium and $1.38 \mathrm{Mb}$ in rice, and involved 219 and 328 putative genes, respectively [5]. Nevertheless, the bin FL0.45-0.58 is still a large chromosomal segment and insufficient for fine mapping Pm21. Thus, it remains difficult to physically map Pm21 further. 
In 2013, two small alien translocation lines NAU418 and NAU419 resistant to powdery mildew were developed by irradiation, both of which carry relatively short segments conveying Pm21 [6]. Moreover, a susceptible deletion line Y18-S6 was recently obtained from ethyl methanesulfonate (EMS)-induced Yangmai 18, a wheat variety carrying translocated chromosome T6AL.6VS [7]. All of them are potential genetic materials for more fine physical mapping of Pm21. In this study, we attempted to physically map Pm21 based on the above genetic stocks. The data obtained here will give a new insight into the chromosome localization of Pm21.

\section{Results}

\subsection{Physical Mapping of Pm21}

In the present study, a total of 45 gene-derived 6VS markers were used to map Pm21. Among them, 42 markers were reported previously, including 6VS-01-6VS-30 [5], 6VS-381 [8], MBH1 [3], Xcinau188, Xcfe164 [9], Xcau127 [10], CINAU15m, 6VS-08.4, 6VS-08.8, 6VS-10.2, 6VS-10.4, 6VS-10.6, and 6VS-10.8 [7]. The other three markers-6VS-08.6, 6VS-12.5, and 6VS-13.5-were newly developed in this study.

The amplification patterns of the representative markers were shown in Figure 1. The results revealed that there are 10 breakpoints on 6VS, designated as b1-b10 according to the direction from the telomere to the centromere (Figure 2, Table 1). Among them, b1 and b10 were in NAU419, b4 and b8 were in NAU418, and b3 and b5 were in Y18-S6. The breakpoints in del.6VS-1 (FL0.58) and del.6VS-1 (FL0.45) were named herein as b2 and b9, respectively. The result showed that b2 and b3 were flanked by the markers 6VS-03 and 6VS-04, and b9 and b10 were flanked by CINAU15m and 6VS-23. Therefore, b2 and b3, and b9 and b10 were close to each other, respectively.

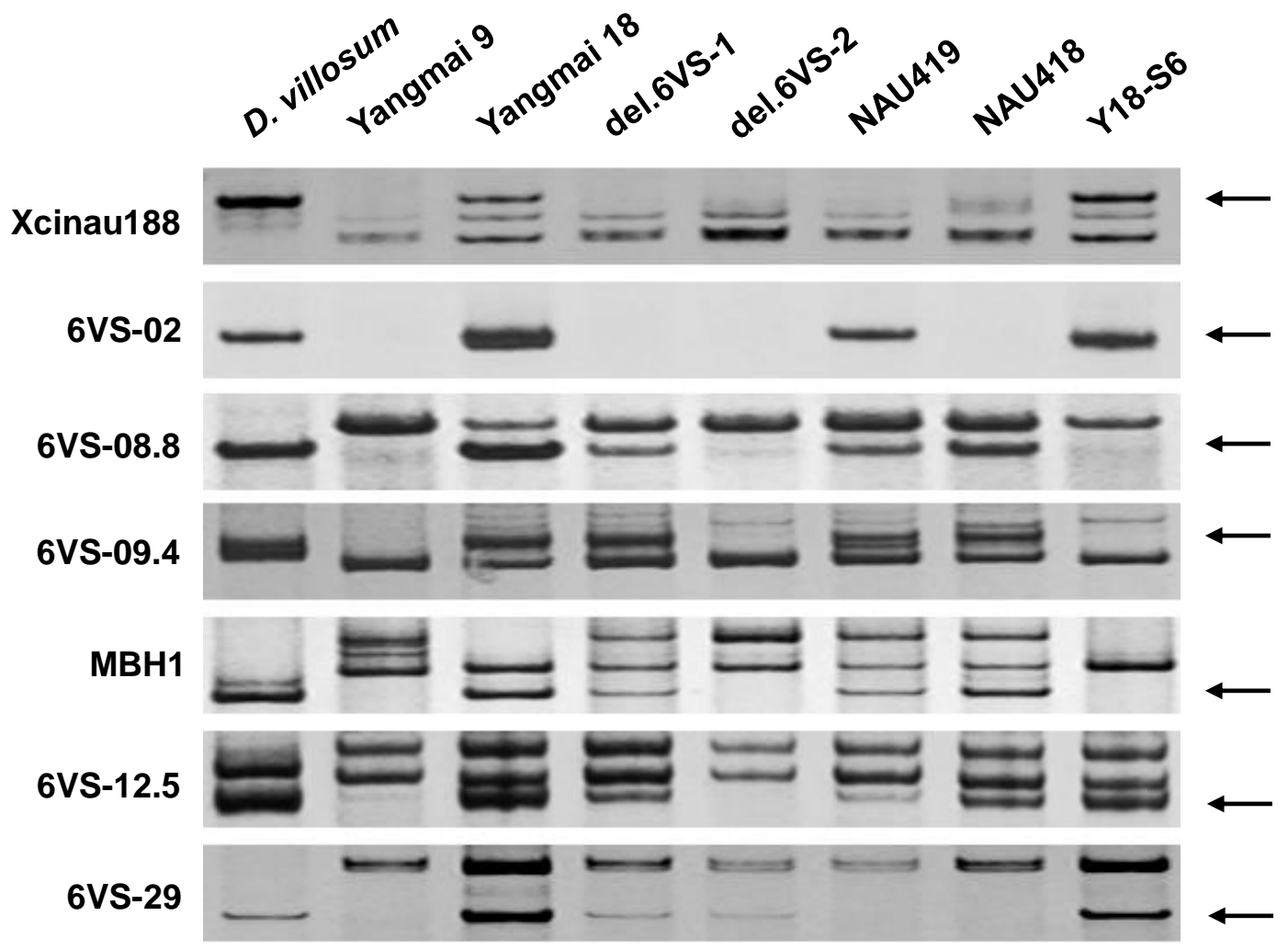

Figure 1. PCR amplification patterns of seven representative markers. The DNA templates used are labeled on the gel, including D. villosum, Yangmai 9, Yangmai 18, del.6VS-1, del.6VS-2, NAU419, NAU418, and Y18-S6. The polymorphic DNA bands are pointed by arrows. 


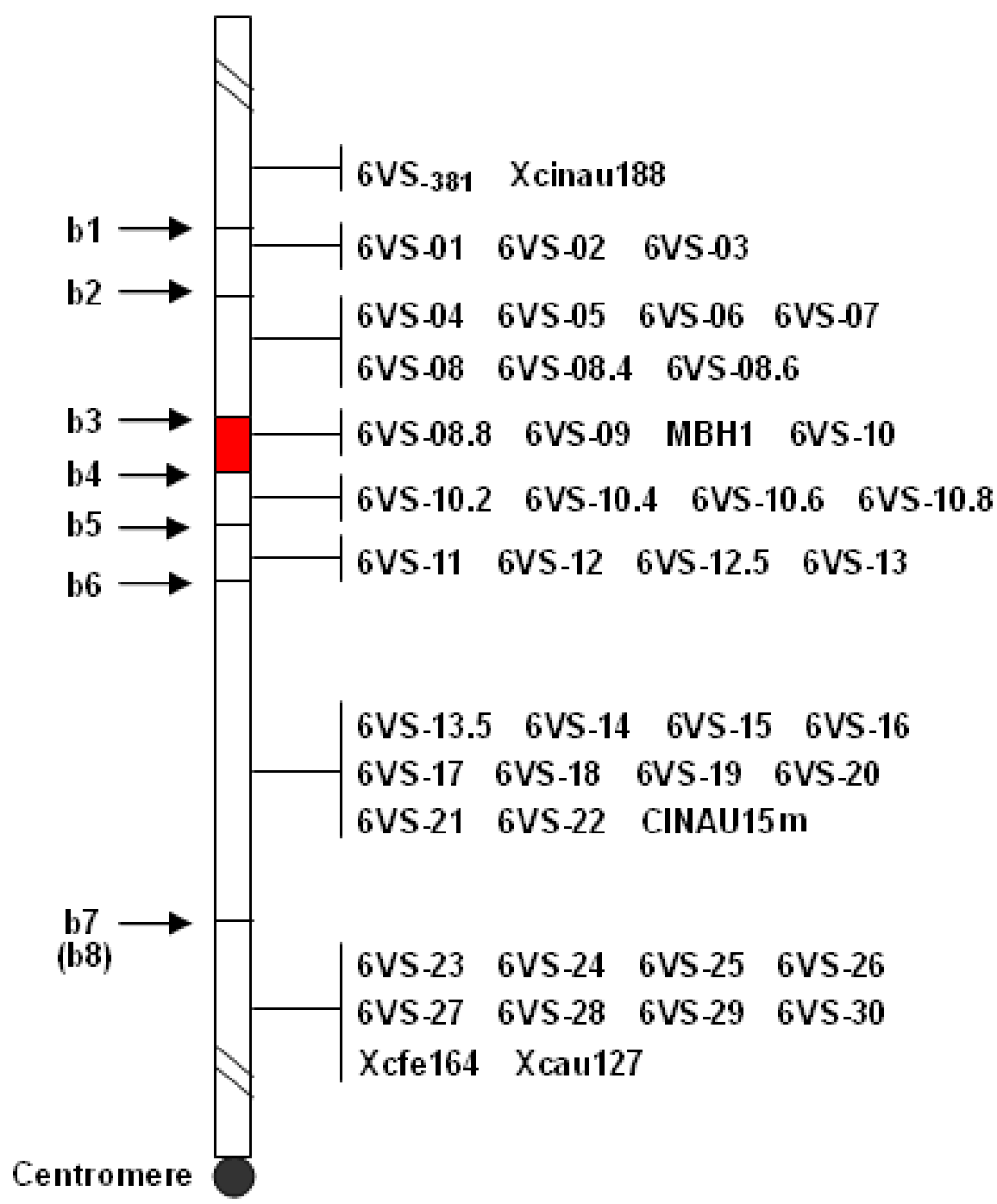

Figure 2. A physical map of 6VS. The breakpoints are shown in the left, and the 6VS-specific DNA markers are shown in the right. The order of the markers is according to the genomic locations of their corresponding orthologous genes in Brachypodium. The red box carries Pm21.

Table 1. Chromosomal breakpoints (b1-b10) in different genetic stocks.

\begin{tabular}{|c|c|c|c|}
\hline Genetic Stock & Treatment & Chromosomal Breakpoint & Flanking Markers \\
\hline \multirow[t]{3}{*}{ del.6VS-1 } & Irradiation $^{a}$ & b2 (FL0.58) & 6VS-03/6VS-04 \\
\hline & & b6 & 6VS-10/6VS-10.2 \\
\hline & & b7 & 6VS-10.8/6VS-11 \\
\hline del.6VS-2 & Irradiation & b9 (FL0.45) & CINAU15m/6VS-23 \\
\hline \multirow{2}{*}{ NAU418 } & Irradiation & b4 & 6VS-08.6/6VS-08.8 \\
\hline & & b8 & 6VS-13/6VS-13.5 \\
\hline \multirow[t]{2}{*}{ NAU419 } & Irradiation & b1 & Xcinau188/6VS-01 \\
\hline & & b10 (Close to b9) & CINAU15m/6VS-23 \\
\hline \multirow[t]{2}{*}{ Y18-S6 } & EMS & b3 (Close to b2) & 6VS-03/6VS-04 \\
\hline & & b5 (Close to b6) & 6VS-10/6VS-10.2 \\
\hline
\end{tabular}

a As described, the deletion line del.6VS-1 was spontaneously formed from the wheat- $D$. villosum addition line DA6V\#2 [11]; however, in fact, it may be obtained after treatment with ${ }^{60} \mathrm{Co} \gamma$-rays (Prof. Peidu Chen, personal communication). 
Unexpectedly, all the polymorphic bands of the markers 6VS-10.2, VS-10.4, 6VS-10.6, and 6VS-10.8 were absent in del.6VS-1 (Figure 3a), which implied that there was an additional deletion in del.6VS-1. Comparative analysis showed that the corresponding genes of the markers 6VS-10.8 and 6VS-11 were all linked on the short arms of chromosome 2 (2OsS) in rice and chromosome 3 (3BdS) in Brachypodium, and on wheat $6 \mathrm{BS}$ and $6 \mathrm{DS}$, except a disruption caused by retrotransposon-like repeat sequence on wheat 6 AS. To detect the linkage relationship of the two genes in $D$. villosum, a pair of primers, P1/P2, was designed according to the conserved sequences between wheat and Brachypodium, and used to amplify the potential fragment. Sequencing analysis showed that the ends of PCR products matched with the corresponding genes of 6VS-10.8 and 6VS-11, respectively (Figure 3b), demonstrating that the corresponding genes of the two markers are also physically linked in D. villosum. Taken together, a new interstitial deletion on 6VS was confirmed in del.6VS-1, and the corresponding breakpoints were then designated as b6 (close to b5) and b7.

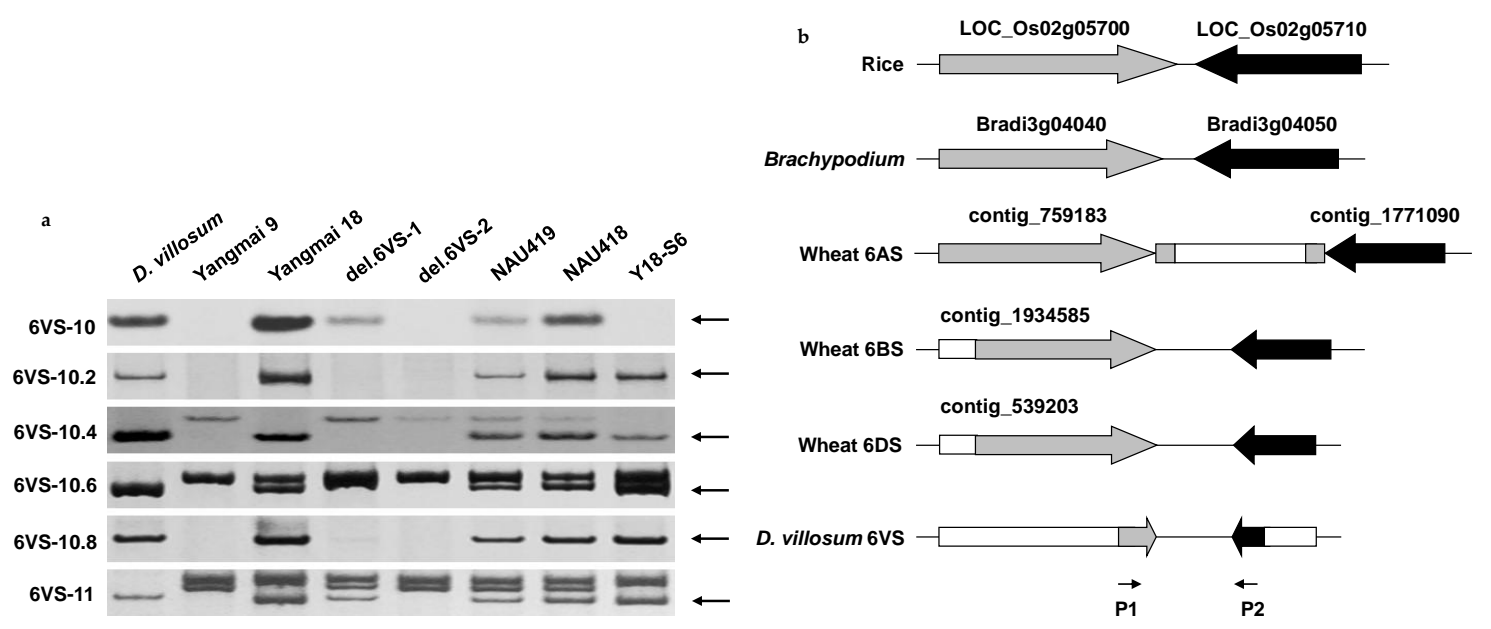

Figure 3. A newfound deletion region of 6VS in del.6VS-1. (a) Amplification patterns of the markers 6VS-10, 6VS-10.2, 6VS-10.4, 6VS-10.6, 6VS-10.8, and 6VS-11. The polymorphic DNA bands are pointed by arrows. All the markers 6VS-10.2, 6VS-10.4, 6VS-10.6 and 6VS-10.8 in the bin FLb5-b6, flanked by 6VS-10 and 6VS-11, were deleted in del.6VS-1; (b) Linkage relationship between the markers 6VS-10.8 and 6VS-11 in different (sub) genomes. The corresponding orthologous genes of 6VS-10.8 and 6VS-11 are indicated by grey and black arrows, respectively. The unknown sequences are shown in white boxes. On wheat 6AS, the grey boxes at the ends of contig_759183 and contig_1771090 indicate retrotransposon-like repeat sequences of wheat genome. On D. villosum 6VS, the linkage relationship of the corresponding genes of 6VS-10.8 and 6VS-11 were detected by PCR using the primers P1 and P2, and then confirmed by sequencing.

Therefore, chromosome 6VS could be divided into eight physical bins, viz., FL0-b9/b10, FLb8-b9/b10, FLb7-b8, FLb5/b6-b7, FLb4-b5/b6, FLb2/b3-b4, FLb1-b2/b3, and FLb1-1.00 (Figure 2). Because both NAU418 carrying the region FLb4-b8 and del.6VS-1 lacking the bin FLb5/b6-b7 are still immune to powdery mildew, Pm21 can be mapped to the small bin FLb4-b5/b6 or FLb7-b8. Given that the deletion line Y18-S6 lacking of the bin FLb3-b5 is susceptible to powdery mildew, it was concluded that $P m 21$ is located on the bin FLb4-b5/b6.

Stpk- $V$, encoding a serine/threonine protein kinase, was previously reported to be a key member of the Pm21 locus [2]. However, this study showed that the dominant marker PK-F2/PK-R [12] and the co-dominant markers CINAU15 [13] and CINAU15m, each of them derived from Stpk-V, together with the flanking markers 6VS-22 and 6VS-23, were all assigned to FLb8-b9/b10, rather than FLb4-b8 which appears in NAU418 (Figures 2 and 4). 


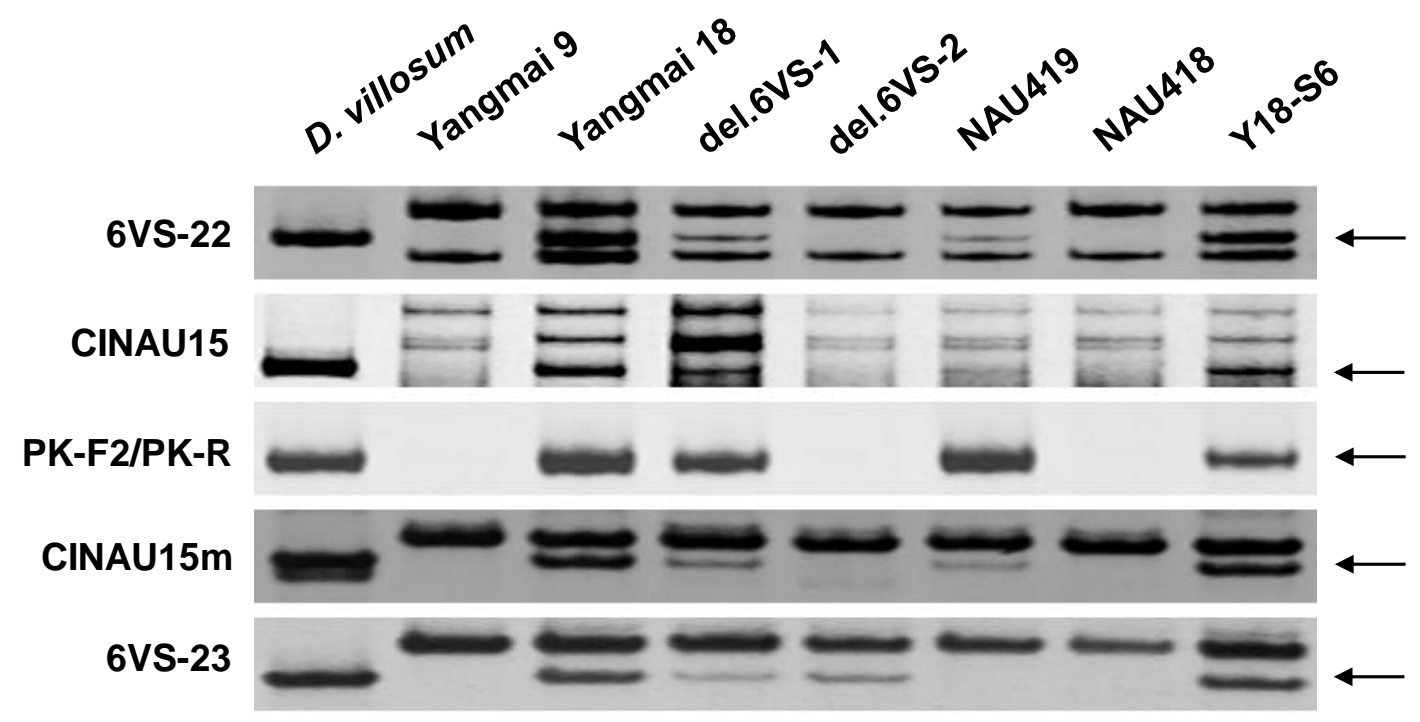

Figure 4. PCR amplification patterns of the markers 6VS-22, CINAU15, PK-F2/PK-R, CINAU15m, and 6VS-23. The polymorphic DNA bands are indicated by arrows.

\subsection{Comparative Genomics Analysis of 6 VS FLb4-b5/b6}

The physical bin FLb4-b5/b6 carrying Pm21 was flanked by the markers 6VS-08.6 and 6VS-10.2. The orthologs of the corresponding genes of 6VS-08.6 and 6VS-10.2 were Bradi3g03850 $(2,587,675-2,590,972)$ and Bradi3g04010 $(2,711,658-2,714,402)$ in Brachypodium, respectively.

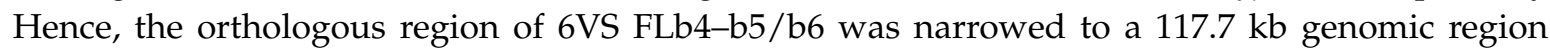
harboring 19 genes in Brachypodium. In rice, due to having no ortholog of 6VS-08.6 in the target region, the region between the corresponding genes LOC_Os02g05610 $(2,728,848-2,734,454)$ and LOC_Os02g05670 $(2,774,720-2,772,143)$ of the markers 6VS-08.4 and 6VS-10.2 was considered as the orthologous region, which is $37.7 \mathrm{~kb}$ in length harboring five genes.

In the orthologous regions of 6VS FLb4-b5/b6, Brachypodium and rice had four conserved genes, which encode an exocyst complex component EXO70A1, a serine/threonine protein phosphatase 2C (PP2C), a homeobox-leucine zipper protein HOX26, and an DEAD (Asp-Glu-Ala-Asp)-box ATP-dependent RNA helicase (DARH), respectively (Table 2). Among them, except $D A R H$, the other three genes-EXO70A1, PP2C, and HOX26-have their orthologs on the short arms of wheat homoeologous group 6 and have been used to develop the markers 6VS-08.8, 6VS-09, and 6VS-10, respectively (Figure 2).

Recently, a conserved resistance gene analog (RGA) locus between wheat and Brachypodium was confirmed to co-segregate with Pm21 in a genetic mapping population derived from a cross between resistant and susceptible D. villosum lines [7]. In this study, comparative analysis showed that the above RGA locus was still in wheat and Brachypodium orthologous regions of FLb4-b5/b6. Molecular detection using the markers MBH1 and 6VS-09.4 [3,7], both derived from this RGA locus, indicated that it could also be assigned to the bin FLb4-b5/b6 (Figures 1 and 2).

Table 2. Gene annotation in Brachypodium, rice and wheat orthologous regions of the bin FLb4-b5/b6 carrying Pm21.

\begin{tabular}{|c|c|c|c|c|}
\hline Marker & Brachypodium & Rice & Wheat & Gene Annotation \\
\hline 6VS-08.6 & Bradi3g03850 & & $\begin{array}{l}\text { 6AS_contigs_4399884 } \\
\text { 6BS_contigs_2953789 } \\
\text { 6DS_contigs_2117578 }\end{array}$ & Eukaryotic translation initiation factor \\
\hline 6VS-08.8 & Bradi3g03860 & LOC_Os02g05620 & $\begin{array}{l}\text { 6AS_contigs_4431592 } \\
\text { 6BS_contigs_2953283 } \\
\text { 6DS_contigs_2092656 }\end{array}$ & $\begin{array}{l}\text { Exocyst complex subunit } \\
\text { EXO70-like protein }\end{array}$ \\
\hline
\end{tabular}


Table 2. Cont.

\begin{tabular}{|c|c|c|c|c|}
\hline Marker & Brachypodium & Rice & Wheat & Gene Annotation \\
\hline 6VS-09 & Bradi3g03870 & LOC_Os02g05630 & $\begin{array}{l}\text { 6AS_contigs_4363243 } \\
\text { 6BS_contigs_2962596 } \\
\text { 6DS_contigs_2093935 }\end{array}$ & Serine/threonine protein phosphatase $2 \mathrm{C}$ \\
\hline MBH1 & Bradi3g03874 ${ }^{a}$ & & Six contigs ${ }^{a}$ & Disease resistance protein \\
\hline \multirow[t]{13}{*}{ 6VS-09.4 } & Bradi3g03878 a & & Six contigs ${ }^{\text {a }}$ & Disease resistance protein \\
\hline & Bradi3g03882 a & & Six contigs ${ }^{\text {a }}$ & Disease resistance protein \\
\hline & Bradi3g03886 & & & Unknown protein \\
\hline & Bradi3g03890 & & & Unknown protein \\
\hline & Bradi3g03900 & & & Unknown protein \\
\hline & Bradi3g03910 & & & Cytochrome P450 \\
\hline & Bradi3g03920 & & & Unknown protein \\
\hline & Bradi3g03930 & & & Poly(A) polymerase \\
\hline & Bradi3g03935 a & & Six contigs ${ }^{\text {a }}$ & Disease resistance protein \\
\hline & Bradi3g03940 & & $\begin{array}{l}\text { 6AS_contigs_4428294 } \\
\text { 6BS_contigs_2926507 } \\
\text { 6DS_contigs_2114667 }\end{array}$ & Photosystem II protein J \\
\hline & Bradi3g03945 & & $\begin{array}{l}\text { 6AS_contigs_4428294 } \\
\text { 6BS_contigs_2926507 } \\
\text { 6DS_contigs_2114667 }\end{array}$ & $\begin{array}{l}\text { Photosystem II cytochrome b559 alpha } \\
\text { subunit }\end{array}$ \\
\hline & Bradi3g03950 & & & Unknown protein \\
\hline & Bradi3g03957 & & $\begin{array}{l}\text { 6AS_contigs_4431958 } \\
\text { 6BS_contigs_2953283 } \\
\text { 6DS_contigs_2081863 }\end{array}$ & Polyubiquitin \\
\hline \multirow[t]{6}{*}{ 6VS-10 } & Bradi3g03970 & LOC_Os02g05640 & $\begin{array}{l}\text { 6AS_contigs_4429249 } \mathrm{b} \\
\text { 6BS_contigs_1424756 b }\end{array}$ & Homeobox-leucine zipper protein HOX26 \\
\hline & & & 6DS_contigs_2087435 b & \\
\hline & & LOC_Os02g05650 & & Unknown protein \\
\hline & Bradi3g03980 & & 6AS_contigs_2062052 & Unknown protein \\
\hline & & & 6DS_contigs_2110435 & \\
\hline & Bradi3g03990 & LOC_Os02g05660 & & DEAD-box ATP-dependent RNA helicase \\
\hline 6VS-10.2 & Bradi3g04000 & LOC_Os02g05661 & $\begin{array}{l}\text { 6AS_contigs_4428740 } \\
\text { 6DS_contigs_2082952 }\end{array}$ & Unknown protein \\
\hline
\end{tabular}

a Four RGAs (Bradi3g03874, Bradi3g03878, Bradi3g03882 and Bradi3g03935) in Brachypodium are highly homologous with six contigs (6AS_contigs_4363243, 6AS_contigs_4428238, 6BS_contigs_3017519, 6BS_contigs_2958212, 6DS_contigs_2091261 and 6DS_contigs_1402211) on the short arm of wheat homologous group 6; ${ }^{-}$Multiple contigs were matched on the short arms of wheat homologous group 6 .

\section{Discussion}

Pm21, one of most effective powdery mildew resistance genes, is highly resistant to all known $B g t$ isolations. However, due to the lack of recombination between the alien chromosome 6VS and wheat homoeologous chromosomes, it is infeasible to genetically map Pm21 in wheat background. In the past, several genetic stocks involving in chromosomal structural changes of 6VS have been reported $[4,6,7,11]$, which allows to physically map Pm21 in common wheat. In the present study, the structural variations in these genetic stocks were scanned by $456 \mathrm{VS}$-specific markers, and a total of 10 chromosomal breakpoints were found. Then, chromosome 6VS was divided into 8 distinguishable physical bins, and Pm21 was finally physically mapped to the bin FLb4-b5/b6, flanked by the markers 6VS-08.6 and 6VS-10.2. In our recent work, the genetic interval carrying Pm21 was confirmed to be flanked by the markers 6VS-08.4b and 6VS-10b [7]. Consequently, the physical location of Pm21 is approximately consistent with the genetic interval.

NAU418 and NAU419 are two small alien translocation lines resistant to powdery mildew [6]. Here, the results indicated that NAU418 carries the bin FLb4-b8, the size of which corresponds to 224.7 $\mathrm{kb}$ (Bradi3g03860-Bradi3g04140) in Brachypodium and 142.1 kb (LOC_Os02g05620-LOC_Os02g05840) in rice, respectively, while NAU419 contains the bin FLb1-b9/b10, almost covering the bin FL0.45-0.58 defined previously [2,4]. Hence, NAU418 carries smaller alien chromosomal segment than NAU419, 
suggesting that NAU418 might have more important value for wheat breeding. Chen et al. reported that the marker CINAU15 derived from Stpk-V, which was confirmed to be a key member of the Pm21 locus since whose overexpression provided high resistance to powdery mildew in transgenic wheat, also appeared in NAU418 [6]. However, in this study, it was found that Stpk-V is not located in NAU418 by molecular analysis. The confusion may be caused by low resolution of the corresponding marker CINAU15, which is subject to false positive detection.

In the past few decades, several powdery mildew resistance genes-such as Pm12, Pm13, and Pm20 [14-16] - have been transferred from wild relatives into common wheat; However, like 6VS carrying Pm21, the corresponding translocated chromosomal arms, 6SS, 3S $\mathrm{S}$, and $6 \mathrm{RL}$, cannot recombine with the ones of wheat. Hence, till now, these genes have not been fine mapped by using classic genetic mapping strategy in wheat background, which hampers the cloning and utilization of these important genes. This study demonstrated that physical mapping using genetic stocks involving in chromosomal structural variations could be an alternative and efficient method for locating these alien genes.

\section{Materials and Methods}

\subsection{Plant Materials}

The common wheat varieties Yangmai 9 and Yangmai 18 were developed in Yangzhou Academy of Agricultural Sciences (YAAS). Yangmai 9 is highly susceptible to powdery mildew. Yangmai 18 carrying a pair of T6AL.6VS is immune to powdery mildew. The D. villosum accession and its derived resistant translocation lines NAU418 and NAU419 [6], resistant deletion lines del.6VS-1 (FL0.58) [11] and susceptible del.6VS-2 (FL0.45) [4] were kindly provided by Professor Peidu Chen, the Cytogenetics Institute, Nanjing Agricultural University. The susceptible deletion line Y18-S6 was obtained from Yangmai 18 mutagenized with EMS in our group [7].

\subsection{Development of 6 VS-Specific Markers}

6VS-specific markers were published in previous works or newly developed here using the CISP (conserved-intron scanning primers) and CISP-IS (CISP combined with intron sequencing) strategies based on the collinearity relationship between Brachypodium, rice, and Triticeae species [5,17]. The newly developed markers were listed in Table 3.

Table 3. 6VS-specific markers newly developed in this study.

\begin{tabular}{ccccc}
\hline Marker & Strategy & Primer Sequence $\left(5^{\prime} \rightarrow \mathbf{3}^{\prime}\right)$ & $\begin{array}{c}\text { Annealing } \\
\text { Temperature }\left({ }^{\circ} \mathbf{C}\right)\end{array}$ & $\begin{array}{c}\text { Size of Polymorphic } \\
\text { Band (bp) }\end{array}$ \\
\hline 6VS-08.6 & CISP-IS & $\begin{array}{c}\text { F: GATCTCGTTTTAGTCCTGAGCCTGT } \\
\text { R: CCGAGTGAAGTCTGCTCATCCAGT }\end{array}$ & 60 & 604 \\
\hline 6VS-12.5 & CISP & $\begin{array}{c}\text { F: CCGGCGACGCGCACTAC } \\
\text { R: GTACTTGTCCGCGAACTCGAAC }\end{array}$ & 60 & 391 \\
\hline 6VS-13.5 & CISP & $\begin{array}{c}\text { F: TGCCTCATCACCCCAGTGAA } \\
\text { R: TGCAGTAGGTGGCATAGAGCAAG }\end{array}$ & 60 & 287 \\
\hline
\end{tabular}

F: forward primer; R: reverse primer.

\subsection{DNA Extraction and PCR}

Genomic DNA was isolated from fresh leaves of the seedlings by the CTAB (cetyl trimethyl ammonium bromide) method [18]. PCR amplification was performed in Peltier thermal cycler (Bio-Rad, Hercules, CA, USA) in $25 \mu \mathrm{L}$ volume containing $1 \times$ PCR buffer, $0.2 \mathrm{mM}$ of each dNTP, $2 \mu \mathrm{M}$ of each primer, 1 Unit of Taq DNA polymerase, and $50 \mathrm{ng}$ genomic DNA. PCR was carried out with an initial denaturation at $94{ }^{\circ} \mathrm{C}$ for $3 \mathrm{~min}, 35$ cycles of $20 \mathrm{~s}$ at $94{ }^{\circ} \mathrm{C}, 30 \mathrm{~s}$ at $60^{\circ} \mathrm{C}, 1 \mathrm{~min}$ at $72{ }^{\circ} \mathrm{C}$, 
and a final extension for $5 \mathrm{~min}$ at $72{ }^{\circ} \mathrm{C}$. PCR products were separated in $6 \%$ or $8 \%$ non-denaturing polyacrylamide gels, silver stained, and photographed.

\subsection{Physical Mapping of Pm21}

Using NAU418, NAU419, del.6VS-1, del.6VS-2, and Y18-S6 involving in structural variation of 6VS as materials, polymorphic DNA markers were assigned to different 6VS bins, and then a high-density physical map was obtained.

\subsection{Comparative Genomics Analysis of the Pm21 Locus}

The genome sequences of Brachypodium, rice and wheat were obtained from the Brachypodium distachyon genome assemblies v2.0 (http:/ / www.brachypodium.org), the rice genome pseudomolecule release 7 (http:/ / rice.plantbiology.msu.edu) and the IWGSC (The International Wheat Genome Sequencing Consortium) Sequence Repository (http://wheat-urgi.versailles.inra.fr), respectively. Genes were predicted by using the FGENESH tool (http:/ /linux1.softberry.com), and then re-annotated by using the BLAST program (http://blast.ncbi.nlm.nih.gov/Blast.cgi) and the SMART program (http://smart.embl-heidelberg.de).

Acknowledgments: This research was supported by Grants from the National Natural Science Foundation of China (31471497, U1604116), the Natural Science Foundation of Jiangsu Province (BK20130503, BK20151319), the Innovation Foundation of Jiangsu Academy of Agricultural Sciences (ZX(17)2011), the Key Research Project of Colleges of Henan Province (15A210017) and the Foundation of Jiangsu University (13JDG103). The authors are grateful to Peidu Chen (the Cytogenetics Institute, Nanjing Agricultural University) for kindly providing the genetic stocks.

Author Contributions: Huagang He and Anli Gao conceived and designed the experiments. Shanying Zhu performed physical mapping, analyzed the data, and wrote the paper. Yaoyong Ji and Jian Ji developed DNA markers. Tongde Bie carried out comparative genomics analysis. All authors commented and approved the final version.

Conflicts of Interest: The authors declare no conflict of interest.

\section{References}

1. Chen, P.D.; Qi, L.L.; Zhou, B.; Zhang, S.Z.; Liu, D.J. Development and molecular cytogenetic analysis of wheat-Haynaldia villosa 6VS/6AL translocation lines specifying resistance to powdery mildew. Theor. Appl. Genet. 1995, 91, 1125-1128. [CrossRef] [PubMed]

2. Cao, A.; Xing, L.; Wang, X.; Yang, X.; Wang, W.; Sun, Y.; Qian, C.; Ni, J.; Chen, Y.; Liu, D.; et al. Serine/threonine kinase gene $S t p k-V$, a key member of powdery mildew resistance gene Pm21, confers powdery mildew resistance in wheat. Proc. Natl. Acad. Sci. USA 2011, 108, 7727-7732. [CrossRef] [PubMed]

3. Bie, T.; Zhao, R.; Zhu, S.; Chen, S.; Cen, B.; Zhang, B.; Gao, D.; Jiang, Z.; Chen, T.; Wang, L.; et al. Development and characterization of marker $\mathrm{MBH} 1$ simultaneously tagging genes $P m 21$ and $P m V$ conferring resistance to powdery mildew in wheat. Mol. Breed. 2015, 35, 189. [CrossRef]

4. Chen, S.; Chen, P.; Wang, X. Inducement of chromosome translocation with small alien segments by irradiating mature female gametes of the whole arm translocation line. Sci. China Ser. C 2008, 51, 346-352. [CrossRef] [PubMed]

5. He, H.; Zhu, S.; Jiang, Z.; Ji, Y.; Wang, F.; Zhao, R.; Bie, T. Comparative mapping of powdery mildew resistance gene Pm21 and functional characterization of resistance-related genes in wheat. Theor. Appl. Genet. 2016, 129, 819-829. [CrossRef] [PubMed]

6. Chen, P.; You, C.; Hu, Y.; Chen, S.; Zhou, B.; Cao, A.; Wang, X. Radiation-induced translocations with reduced Haynaldia villosa chromatin at the Pm21 locus for powdery mildew resistance in wheat. Mol. Breed. 2013, 31, 477-484. [CrossRef]

7. He, H.; Ji, Y.; Zhu, S.; Li, B.; Zhao, R.; Jiang, Z.; Bie, T. Genetic, physical and comparative mapping of the powdery mildew resistance gene Pm21 originating from Dasypyrum villosum. Front. Plant Sci. 2017, 8, 1914. [CrossRef] [PubMed] 
8. Bie, T.; Zhao, R.; Jiang, Z.; Gao, D.; Zhang, B.; He, H. Efficient marker-assisted screening of structural changes involving Haynaldia villosa chromosome 6V using a double-distal-marker strategy. Mol. Breed. 2015, 35, 34. [CrossRef]

9. Qi, X.; Cui, F.; Li, Y.; Ding, A.; Li, J.; Chen, G.; Wang, H. Molecular tagging wheat powdery mildew resistance gene Pm 21 by EST-SSR and STS markers. Mol. Plant Breed. 2010, 1, 22-26. [CrossRef]

10. Song, W.; Xie, C.; Du, J.; Xie, H.; Liu, Q.; Ni, Z.; Yang, T.; Sun, Q.; Liu, Z. A “one-marker-for-two-genes" approach for efficient molecular discrimination of $P m 12$ and $P m 21$ conferring resistance to powdery mildew in wheat. Mol. Breed. 2009, 23, 357-363. [CrossRef]

11. Qi, L.L.; Wang, S.L.; Chen, P.D.; Liu, D.J.; Gill, B.S. Identification and physical mapping of three Haynaldia villosa chromosome-6V deletion lines. Theor. Appl. Genet. 1998, 97, 1042-1046. [CrossRef]

12. Zhang, Y.; Wang, M.; Zhang, Y.; Chu, C.; Lin, Z.; Xu, Q.; Ye, X.; Chen, X.; Zhang, X. Development and application of functional markers specific to powdery mildew resistance on chromosome arm 6VS from different origins of Haynaldia villosa. Acta Agron. Sin. 2012, 38, 1827-1832. [CrossRef]

13. Cao, A.Z.; Wang, X.E.; Chen, Y.P.; Zou, X.W.; Chen, P.D. A sequence-specific PCR marker linked with Pm21 distinguishes chromosomes 6AS, 6BS, 6DS of Triticum aestivum and 6VS of Haynaldia villosa. Plant Breed. 2006, 125, 201-205. [CrossRef]

14. Friebe, B.; Heun, M.; Tuleen, N.; Zeller, F.J.; Gill, B.S. Cytogenetically monitored transfer of powdery mildew resistance from rye into wheat. Crop Sci. 1994, 34, 621-625. [CrossRef]

15. Jia, J.; Devos, K.M.; Chao, S.; Miller, T.E.; Reader, S.M.; Gale, M.D. RFLP-based maps of the homoeologous group-6 chromosomes of wheat and their application in the tagging of $P m 12$, a powdery mildew resistance gene transferred from Aegilops speltoides to wheat. Theor. Appl. Genet. 1996, 92, 559-565. [CrossRef] [PubMed]

16. Cenci, A.; D'Ovidio, R.; Tanzarella, O.A.; Ceoloni, C.; Porceddu, E. Identification of molecular markers linked to Pm13, an Aegilops longissima gene conferring resistance to powdery mildew in wheat. Theor. Appl. Genet. 1999, 98, 448-454. [CrossRef]

17. He, H.; Zhu, S.; Sun, W.; Gao, D.; Bie, T. Efficient development of Haynaldia villosa chromosome 6VS-specific DNA markers using a CISP-IS strategy. Plant Breed. 2013, 132, 290-294. [CrossRef]

18. Murray, M.G.; Thompson, W.F. Rapid isolation of high molecular weight plant DNA. Nucleic Acids Res. 1980, 8, 4321-4325. [CrossRef] [PubMed] 